\title{
Overexpression of COUP-TFII suppresses proliferation and metastasis of human gastric cancer cells
}

\author{
WEIJI DING ${ }^{1,2^{*}}$, YUNDA ZHANG $^{1,3^{*}}$, HUALI CAI $^{1,2^{*}}$, GANG LIU $^{1,3}$, YONGZHI YE $^{1}$, GUOXING XU $^{1,2}$, \\ HAIBIN WANG $^{1,2}$, DISHENG XIONG ${ }^{1,2}$, CHUANKAI ZHANG ${ }^{1,3}$, ZHENGJIE HUANG ${ }^{1,2}$ and QI LUO ${ }^{1,2}$
}

\author{
${ }^{1}$ Department of Gastrointestinal Surgery, Xiamen Cancer Hospital, The First Affiliated Hospital of Xiamen University, \\ Xiamen, Fujian 361003; ${ }^{2}$ Department of Gastrointestinal Surgery, First Clinical Medical College of Fujian Medical University, \\ Fuzhou, Fujian 350004; ${ }^{3}$ State Key Laboratory of Cellular Stress Biology, Innovation Center for Cell Signaling Network, \\ School of Life Sciences, Xiamen University, Xiamen, Fujian 361102, P.R. China
}

Received February 28, 2017; Accepted October 4, 2017

DOI: $10.3892 / \mathrm{mmr} .2017 .8164$

\begin{abstract}
The abnormal expression of the chicken ovalbumin upstream promoter transcription factor 2 (COUP-TFII) is associated with numerous forms of cancer, including gastric, prostate, colon and lung cancer. However, previous studies investigating the association between COUP-TFII expression and the occurrence, recurrence, invasion and metastasis of gastric cancer are limited in number. In the present study, it was revealed that the expression of COUP-TFII is significantly reduced in gastric carcinoma tissues compared with normal gastric mucosa cells (GES-1). In addition, the expression of COUP-TFII was also reduced in gastric cancer cell lines compared with GES-1 cells. Furthermore, it was revealed that ectopic expression of COUP-TFII was able to suppress the proliferation, migration and invasion of gastric cells, as well as inhibit hepatic metastasis, in vivo. In addition, it was demonstrated that COUP-TFII knockdown was able to promote the proliferation, migration and invasion of GES-1 cells in vitro. Furthermore, database analysis suggested that COUP-TFII expression in patients with gastric cancer is correlated with clinical stage classification and increased expression levels of COUP-TFII improved overall survival rates in patients with gastric cancer. The results of the present study suggest that COUP-TFII functions as a significant regulatory suppressor of gastric cancer growth and metastasis, and suggests that
\end{abstract}

Correspondence to: Dr Zhengjie Huang or Dr Qi Luo, Department of Gastrointestinal Surgery, Xiamen Cancer Hospital, The First Affiliated Hospital of Xiamen University, 55 Zhen Hai Road, Xiamen, Fujian 361003, P.R. China

E-mail: huangzhengjie@xmu.edu.cn

E-mail: luoqi@xmu.edu.cn

${ }^{*}$ Contributed equally

Key words: gastric cancer, chicken ovalbumin upstream promoter transcription factor 2, proliferation, metastasis
COUP-TFII may serve as a novel diagnostic and prognostic biomarker for gastric cancer metastasis.

\section{Introduction}

Gastric cancer accounts for $9.7 \%$ of the total number of cancer-associated mortalities globally, and accounts for $7.8 \%$ of new malignant tumor diagnoses annually (1). Gastroscopy is one of the early diagnosis methods in gastric cancer, and the 5-year survival rate of patients with gastric cancer has been improved due to gastroscopy (2). The early recurrence of gastric cancer resulting from lymphatic vessel micrometastasis, as well as increased tolerance to radiotherapy and chemotherapy in advanced gastric cancer, are predominant risk factors to be addressed in order to improve gastric cancer prognosis $(3,4)$. The lymph node and hematogenous metastasis pathways are the primary metastasis pathways associated with gastric cancer and are closely associated with gastric cancer prognosis. Therefore, it is important to investigate potential therapeutic targets associated with the occurrence and metastasis of gastric cancer, and to explore molecular targeted therapy and prognosis of gastric cancer.

The chicken ovalbumin upstream transcription factors (COUP-TFs), belonging to the steroid/thyroid hormone receptor family, were discovered in 1986 (5-7). The COUP-TFs family members include two highly homologous proteins, termed COUP-TFI $(8,9)$ and COUP-TFII $(10,11)$, and their encoding genes are located on chromosome 5 and 15, respectively. COUP-TFII is generally considered to be an orphan nuclear receptor, as its ligand(s) have not yet been determined. COUP-TFII is also known as nuclear receptor subfamily 2 , group F, member 2. According to previous studies, COUP-TFII is implicated in the regulation of gene expression and organism development, cell differentiation and homeostasis, and is also involved in tumorigenesis and metastasis processes (12-14). COUP-TFII expression levels are associated with tumorigenesis, invasion and metastasis in numerous cancer types, such as breast, prostate (12), pancreatic (15), ovarian (16) and colorectal cancer (17). However, the association between COUP-TFII and gastric cancer has not yet been fully determined. This study aimed to investigate the potential use of COUP-TFII as a 
gastric cancer biomarker, and its use as a molecular target in targeted therapy. Further study of the COUP-TFII protein may provide novel alternatives to the current therapeutic treatment options available to patients with gastric cancer.

\section{Materials and methods}

Clinical tissue samples and cell lines. Gastric cancer tissues and their matched normal adjacent tissues were obtained from the Department of Gastrointestinal Surgery, The First Affiliated Hospital of Xiamen University (Xiamen, China), between September 2013 to February 2016. The patients' age ranged from 39 to 76-years-old with a distribution of $57.9 \%$ of males among the patients. All samples were collected with patients' informed consent, and all tissues were pathologically confirmed. This study was approved by the Ethics Committee of the First Affiliated Hospital of Xiamen University (Xiamen, China). In the present study, tumor staging criteria were based on the 2010 American Joint Committee on Cancer's TNM staging criteria for gastric cancer (18). BGC-823, MGC-803 and SGC-7901 gastric cancer cells, GES-1 normal gastric mucosal epithelial cells and $293 \mathrm{~T}$ cells, were obtained from the Institute of Biochemistry and Cell Biology, Chinese Academy of Sciences (Shanghai, China). All gastric cancer cells and GES-1 cells were maintained in RPMI1640 media (Sigma-Aldrich; Merck KGaA, Darmstadt, Germany) (19), whereas the 293T cells were cultured in Dulbecco's modified Eagle's medium (Sigma-Aldrich; Merck KGaA) supplemented with $10 \%$ fetal bovine serum (FBS, Gibco; Thermo Fisher Scientific, Inc., Waltham, MA, USA). All cells were maintained at $37^{\circ} \mathrm{C}$ in a humidified incubator with $5 \% \mathrm{CO}_{2}$.

Plasmid construction and generation of stable cell lines. COUP-TFII was amplified using cDNA and then inserted into the pCDH-CMV-MCS-EF1-Puro plasmid (System Biosciences, Palo Alto, CA, USA). The primers used to generate these constructs were as follows: COUP-TFII forward, 5'-GCTCTA GAATGGCAATGGTAGTCAGCACGT-3' and reverse, 5'-ATA AGAATGCGGCCGCTTATTGAATTGCCATATACGGCC AGT-3'; GAPDH (control) forward, 5'-GTGGACCTGACC TGCCGTCT-3', and reverse, 5'-GGAGGAGTGGGTGTCGCT GT-3'. For the generation of COUP-TFII stable cell lines, a lentivirus-mediated packaging system containing four plasmids, COUP-TFII or control plasmid (System Biosciences), pMDL, REV and VSVG (Invitrogen; Thermo Fisher Scientific, Inc.) were used as described (20). For the stable knockdown COUP-TFII in GES-1 cells, pLL3.7-puro containing COUP-TFII small interfering RNA (siRNA) or negative control was co-transfected with pMDL, REV and VSVG as described (20). The siRNA sequences were as follows: siRNA\#1 forward, 5'-GCGAGC UGUUUGUGUUGAATT-3' and reverse, 5'-UUCAACACA AACAGCUCGCTT-3'; siRNA\#2 forward, 5'-GGAUCUUCC AAGAGCAAGUTT-3' and reverse, 5'-ACUUGCUCUUGG AAGAUCCTT-3'; siRNA\#3 forward, 5'-GGCCGUAUAUGG CAAUUCATT-3' and reverse, 5'-UGAAUUGCCAUAUAC GGCCTT-3'. Transfected cells were cultured for $72 \mathrm{~h}$ prior to subsequent experimentation.

Reverse transcription-quantitative polymerase chain reaction $(R T-q P C R)$. Total RNA was extracted from tissue samples and cell lines using the TRIzol reagent (Invitrogen; Thermo Fisher Scientific, Inc.,) according to the manufacturer's instructions. For mRNA reverse transcription, cDNA was synthesized using the ReverTra AceH qPCR RT kit (Toyobo, Osaka, Japan) with $1 \mathrm{mg}$ total RNA. RT-qPCR was performed via SYBR Green assay (Invitrogen; Thermo Fisher Scientific, Inc.) using the SYBRH Select Master Mix for CFX (Invitrogen; Thermo Fisher Scientific, Inc.) as described (21). GAPDH was used as the control. The reverse transcription primers and $\mathrm{qPCR}$ primers of COUP-TFII and GAPDH were purchased from Thermo Fisher Scientific, Inc. COUP-TFII relative expression was evaluated by using the $2^{-\Delta \Delta \mathrm{Cq}}$ method (22). All PCR assays were performed in triplicate.

Cell proliferation assay. In order to determine cell viability, a MTT assay was performed according to a recent study (21). Firstly, MGC-803 and GES-1 cells were seeded and transfected in a 96-well plate. At 24, 48, 72, 96 and $120 \mathrm{~h}$ time intervals post-transfection, MTT $(0.5 \mathrm{mg} / \mathrm{ml}$, Sigma-Aldrich; Merck $\mathrm{KGaA}$ ) was added to the cells, and the absorbance at $490 \mathrm{~nm}$ was then measured $3 \mathrm{~h}$ later. For colony formation assays, cells were seeded in 6-well plates and maintained in RPMI 1640 medium containing for 10-14 days as described (23). Colonies were fixed with $4 \%$ paraformaldehyde and incubated for $15 \mathrm{~min}$ at room temperature, stained with $0.1 \%$ crystal violet for $15 \mathrm{~min}$ at room temperature, and then photographed using a digital camera (23). Experiments were repeated at least three times.

Migration and invasion assays. The Transwell migration assay was performed as described (21). A total of $5 \times 10^{4}$ MGC-803 or GES-1 cells were plated on the top chambers of $8 \mu \mathrm{m}$ pore size Transwell plates (Corning Incorporated, Corning, NY, USA). In order to perform the Matrigel-coated Transwell invasion assay as described (21), Matrigel and $8 \times 10^{4}$ MGC-803 or GES-1 cells were plated on the top chambers of $8 \mu \mathrm{m}$ pore size Transwell plates (Corning Incorporated, Corning, NY, USA). The migration and invasion assays were conducted for $48 \mathrm{~h}$; all experiments were performed at least three times in triplicate.

Western blot analysis. Western blotting was performed as described (21). Cell lysates (10 mg) were subjected to $10 \%$ SDS-PAGE analysis and immunoblotting analysis using antibodies against COUP-TFII (1:5,000; ab64849, Abcam, Cambridge, UK), and either $\beta$-actin (1:5,000; ab8227, Abcam, Cambridge, UK) was used as a protein loading control. Proteins were visualized with an ECL kit and film (Kodak, Rochester, NY, USA) in a dark room; the film was scanned using a HP ScanJet Pro 2000 (Hewlett-Packard, Palo Alto, CA, USA) and analyzed with ImageJ software (v 1.46r; National Institutes of Health, Bethesda, MD, USA).

Immunohistochemistry. Sections were then cut and stained using immunohistochemistry as described (20). Briefly, the sections were deparaffinized, rehydrated by using a descending alcohol series, subjected to microwave antigen retrieval buffer (Sigma-Aldrich; Merck KGaA) and then incubated for $10 \mathrm{~min}$ with $3 \%$ hydrogen peroxide at room temperature. Non-specific binding was blocked using $5 \%$ bovine serum albumin (Sigma-Aldrich; Merck KGaA) for $30 \mathrm{~min}$ at $37^{\circ} \mathrm{C}$. The sections 
were then incubated with rabbit polyclonal anti-COUP-TFII antibody (1:200; ab64849, Abcam) overnight at $4^{\circ} \mathrm{C}$, and then incubated with goat anti-mouse immunoglobulin G H\&L biotinylated secondary antibody (1:200; ab6788, Abcam) bound to a streptavidin-horseradish peroxidase complex for $30 \mathrm{~min}$ in the dark at room temperature. The bound antibody was detected using 3,3'-diaminobenzidine (Sigma-Aldrich; Merck $\mathrm{KGaA}$ ) according to the manufacturer's protocols, and the sections were then counterstained with hematoxylin at room temperature for $1 \mathrm{~min}$, dehydrated and mounted. Two pathologists scored all sections independently. The staining index was calculated as the product of the staining intensity (-, no staining; +, weak, light yellow; ++, moderate, yellow brown; +++, strong, brown). Staining intensity was determined using Image Pro Plus software (version 6.0; Media Cybernetics, Inc., Rockville, MD, USA); the average integrated optical density (IOD) was obtained by analyzing five fields per slide. The average IOD of tumor tissue was divided by the average IOD of paired normal tissue to attain the relative average IOD.

Tumorigenicity in vivo. The Animal Care and Use Committee of Xiamen University approved this study and all its experimental protocols. For tail vein metastasis, either $5 \times 10^{5}$ MGC-803/COUP-TFII cells or $5 \times 10^{5}$ MGC-803 control cells were injected into 6 -week-old male BALB/C mice $(n=4$ per group; weight $=26.3 \pm 2.4 \mathrm{~g}$; provided by the Experimental Animal Center of Xiamen University, Xiamen, China). Hepatic metastasis on day 14 was monitored as described (20) once a week for 4 weeks, using the live animal Lumina II system (Xenogen IVIS system; PerkinElmer, Inc., Waltham, MA, USA).

Database analysis. A dataset with clinical pathological parameters in 80 gastric patients was obtained from the Oncomine database Cui Gastric (3610110; https://www.oncomine.org/); the relevance between COUP-TFII expression level and patients' clinical stage and overall survival was investigated using the analysis of 'Gene summary view' and 'Dataset view' (24,25). Data of COUP TFII expression levels of gastric cancer tissue and relative gastric tissue from a dataset were standardized and compared; if the value obtained was greater within gastric cancerous tissue than the corresponding healthy tissue, expression would be regarded as 'overexpressed'. However, low expression would be observed if the values were lower within gastric cancer tissue compared with in the healthy tissue.

Statistical analysis. SPSS 19.0 software (IBM Corp., Armonk, NY, USA) was used for statistical analysis. All images were made using GraphPad Prism 6 software (GraphPad Software, Inc., La Jolla, CA, USA). Data are presented as mean \pm standard deviation. The two-tailed Student's t-test and one-way analysis of variance, followed by a Chi-square post-hoc test were used in order to determine $\mathrm{P}$-values, and $\mathrm{P}<0.05$ was considered to indicate a statistically significant difference.

\section{Results}

COUP-TFII is downregulated in gastric cancer tissues and gastric cancer cell lines. In order to investigate the difference in COUP-TFII expression between gastric cancer tissues and their matched adjacent non-neoplastic tissues (ANT), the COUP-TFII expression levels were investigated using RT-qPCR and western blot analysis on samples derived from patients with gastric cancer. Subsequently, it was revealed that COUP-TFII mRNA and protein expression levels in gastric carcinoma samples were significantly reduced compared with normal gastric mucosa tissues in 34 cases and 19 cases, respectively (Fig. 1A and B). Furthermore, immunohistochemical analysis revealed that COUP-TFII expression in gastric cancer tissues (11/19) was reduced compared with COUP-TFII expression in ANT (Fig. 1C, scale bar, $200 \mu \mathrm{m}$; Table I). The expression levels of COUP-TFII mRNA and protein in three gastric cancer cell lines (SGC-7901, SGC-823 and MGC-803), and a normal gastric mucosa cell line (GES-1), were then determined via RT-qPCR and western blotting (Fig. 1D). Compared with GES-1, the expression level of COUP-TFII was reduced in all gastric cell lines, particularly in the MGC-803 cell line $(\mathrm{P}<0.001)$. These results therefore suggest that COUP-TFII expression has an association with gastric cancer prognosis.

COUP-TFII inhibits proliferation, migration and invasion of gastric cancer cell in vitro. To determine the effect of COUP-TFII on gastric cancer cell proliferation, a COUP-TFII expression plasmid was transfected into the MGC-803 cell line (Fig. 2A), and cell proliferation was determined via MTT assay. As revealed by Fig. 2B, the level of cellular proliferation in the MGC-803/COUP-TFII group was significantly reduced compared with the MGC-803 and MGC-803 control groups $(\mathrm{P}<0.001)$. In order to determine whether COUP-TFII could modulate the metastasis of gastric cancer cells, Transwell assays were performed to evaluate the migration and invasion capacities of the cells in vitro. This revealed a significant reduction in the migratory and the invasive abilities of the MGC-803/COUP-TFII group ( $\mathrm{P}<0.001$; Fig. 2C and D; scale bar, $500 \mu \mathrm{m})$. In conclusion, these results demonstrate that COUP-TFII expression inhibits proliferation, migration and invasion of gastric cancer cells in vitro.

Downregulated COUP-TFII enhances the proliferative, migratory and invasive abilities of GES-1. As a result of siRNA transfection, three COUP-TFII knockdown cell lines were established (group si\#1, si\#2, si\#3). Western blotting analysis demonstrated that COUP-TFII expression was reduced by the siRNA compared with the control group $(\mathrm{P}<0.01$; Fig. 3A). Furthermore, the MTT assay and the colony formation experiment revealed that knockdown of COUP-TFII in GES-1 cells enhanced their proliferative ability to different extents $(\mathrm{P}<0.05$; Fig. $3 \mathrm{~B}$ and $\mathrm{C}$; scale bar, $6 \mathrm{~mm}$ ). In addition, the results of the Transwell assays revealed that the migratory $(\mathrm{P}<0.01)$ and invasive $(\mathrm{P}<0.001)$ abilities of GES-1 cells were also significantly increased by COUP-TFII knockdown compared with the negative control (Fig. 3D; scale bar, $500 \mu \mathrm{m}$ ).

COUP-TFII inhibits tumor growth and hepatic metastasis of gastric cancer in vivo. In order to determine whether COUP-TFII expression could inhibit tumor growth and metastasis of gastric cancer cells in vivo, MGC-803/COUP-TFII cells and MGC-803 control cells were labeled with 
Table I. COUP-TFII expression in clinical gastric cancer samples and ANT tissues revealed by immunohistochemistry.

Staining index of COUP-TFII expression

\begin{tabular}{lccccc}
\cline { 3 - 5 } Sample & Number & $(-)$ & $(+)$ & $(++)$ & $(+++)$ \\
\hline ANT & 19 & 5 & 1 & 2 & 11 \\
Gastric cancer & 19 & 11 & 1 & 3 & 4
\end{tabular}

ANT, adjacent non-neoplastic tissues; COUP-FFII, chicken ovalbumin upstream promoter transcription factor 2; (-), no staining; (+), weak, light yellow; (++), moderate, yellow brown; (+++), strong, brown.
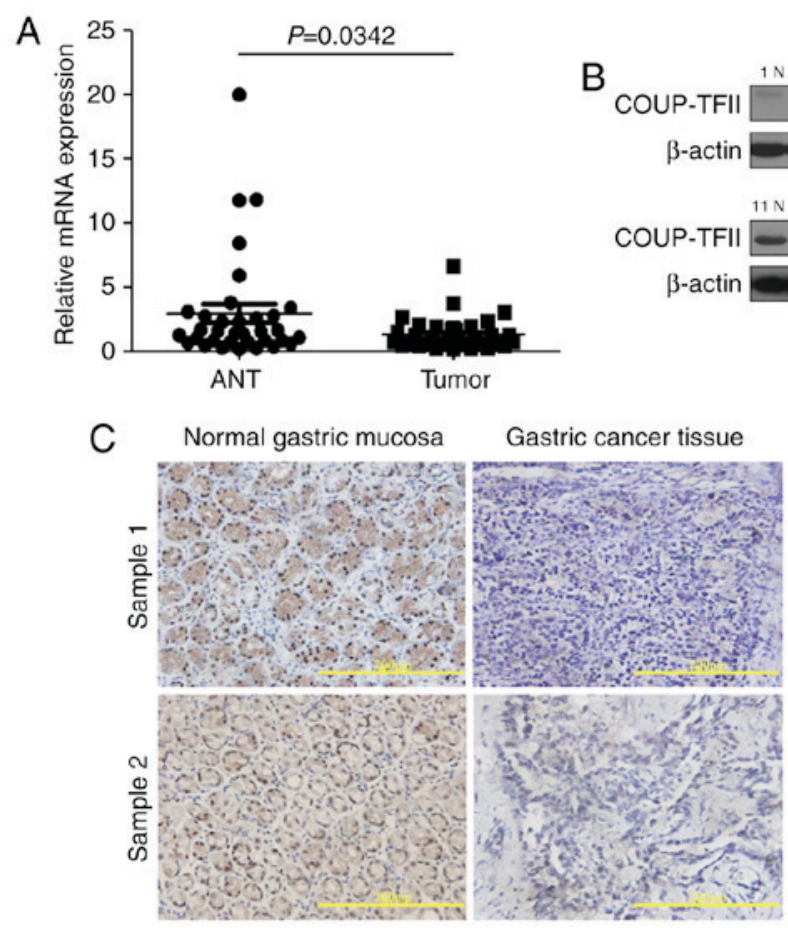

$\beta$-actin

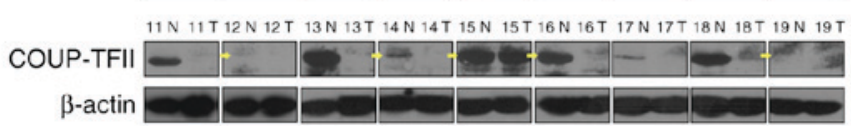

D
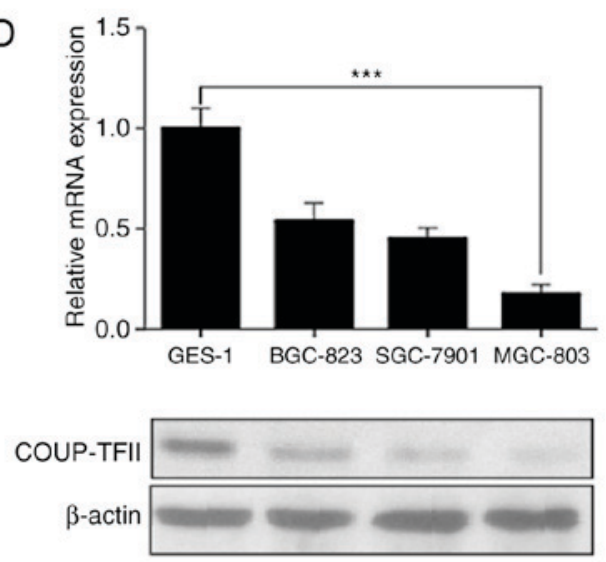

Figure 1. COUP-TFII is downregulated in gastric cancer tissues and gastric cancer cell lines. (A) Difference in the relative expression between all ANT and tumor samples determined by RT-qPCR. (B) Western blotting analysis of 19 cases reveals the differential expression of ANT and tumor samples. Yellow arrows represent the labeling of the correct size of band. (C) Immunohistochemical analysis demonstrates the decreased expression of COUP-TFII in gastric cancer tissues (11/19) compared with normal gastric mucosa tissues; scale bar, $200 \mu \mathrm{m}$. (D) Expression levels of COUP-TFII mRNA and protein in three gastric cancer cell lines (SGC-7901, SGC-823, MGC-803) as well as the GES-1 cell line. ${ }^{* * *} \mathrm{P}<0.001$. ANT, adjacent non-neoplastic tissues; COUP-TFII, chicken ovalbumin upstream promoter transcription factor 2; N, normal; T, tumor.

luciferase, injected into nude mice, and then analyzed 2 weeks later. Bioluminescence imaging results revealed that MGC-803/COUP-TFII cells produced significantly smaller tumors compared with the mice injected with MGC-803 control cells (Fig. 4A). Associated statistical analysis of luminescence intensity is presented in Fig. 4B $(\mathrm{P}<0.01)$. Furthermore, the number of hepatic metastases in the MGC-803/COUP-TFII group was less than in the MGC-803 control group $(\mathrm{P}<0.001$; Fig. 4C and D; scale bar, $100 \mu \mathrm{m}$ ).

Database analysis suggests that high expression level of COUP-TFII is significantly associated with patients' clinical stage and overall survival. As presented in Table II, analysis of the clinical pathological parameters in 80 gastric patients obtained from the Oncomine database (https://www.oncomine.org/) suggested that the expression level of COUP-TFII in gastric cancer has no association with the patient's sex, age and grade $(\mathrm{P}>0.05)$, however, it does have an association with the patient's clinical stage $(\mathrm{P}<0.01$; Table II). The Kaplan-Meier plot revealed that high expression levels of COUP-TFII improved overall survival in patients with gastric cancer (Fig. 5).

\section{Discussion}

Gastric cancer is one of the most prevalent gastrointestinal malignant tumors, and poses a significant threat to health and life expectancy. Well-established treatments, such as surgical procedures, do not always meet the requirements of patients. As a consequence of accumulating research in recent years, molecular targeted therapy is fast becoming an increasingly prominent field within oncology research. Nuclear receptors are significantly implicated in the development of tumors. Nuclear receptors themselves, or their downstream target molecules, 

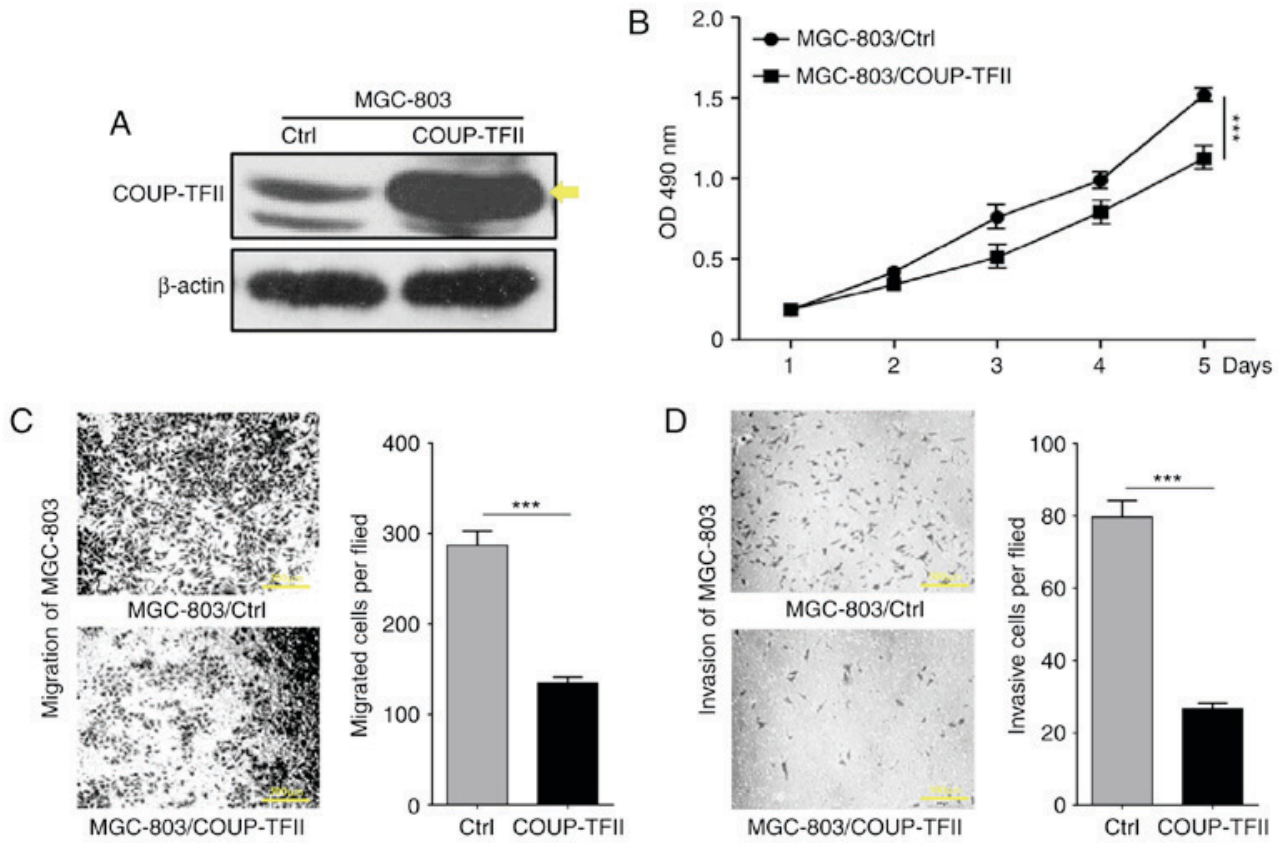

Figure 2. COUP-TFII inhibits proliferation, migration and invasion of gastric cancer cells in vitro. (A) Western blotting analysis reveals that COUP-TFII overexpression in the transfected MGC-803 cell line. Yellow arrows represent the labeling of the correct size of band. (B) MTT assay reveals the effects of COUP-TFII expression on the proliferation of MCG-803 cells. (C) Transwell migration assays and (D) invasion assay with Matrigel-coated membranes of COUP-TFII. Scale bar, $500 \mu \mathrm{m} .{ }^{* * *} \mathrm{P}<0.001$. COUP-TFII, chicken ovalbumin upstream promoter transcription factor 2; OD, optical density.
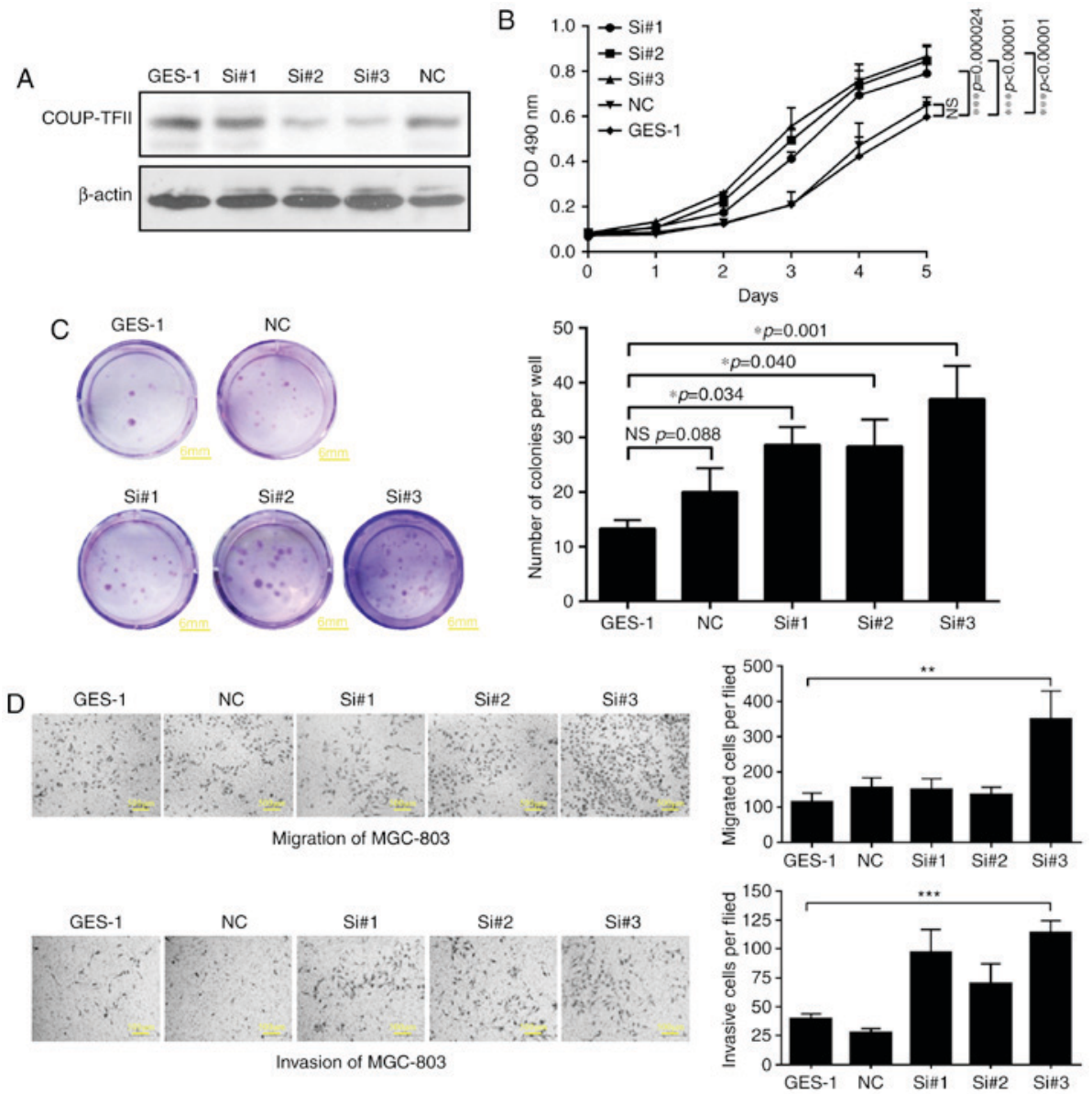

Figure 3. Downregulation of COUP-TFII expression enhances the proliferation, migration and invasion of GES-1 cells. (A) Relative COUP-TFII expression revealed by western blotting analysis in three COUP-TFII knockdown cell lines (groups si\#1, si\#2, and si\#3). (B) MTT assay and the (C) colony formation experiments demonstrate that following downregulation of COUP-TFII gene expression in GES-1 cells, cellular proliferative ability is differentially affected. Scale bar, $6 \mathrm{~mm}$. (D) Transwell migration assays and invasion assays with Matrigel-coated membranes of three COUP-TFII knockdown cell lines. Scale bar, $500 \mu \mathrm{m} .{ }^{~} \mathrm{P}<0.05,{ }^{* *} \mathrm{P}<0.01,{ }^{* * *} \mathrm{P}<0.001$. COUP-TFII, chicken ovalbumin upstream promoter transcription factor 2; NS, no significance; NC, negative control; $\mathrm{Si}$, siRNA. 
Table II. Statistical analysis of gastric cancer clinical sample data from Oncomine database.

\begin{tabular}{|c|c|c|c|c|c|}
\hline Clinical data & Samples (n) & $\begin{array}{c}\text { High } \\
\text { expression of COUP-TFII }(\%)\end{array}$ & $\begin{array}{c}\text { Low } \\
\text { expression of COUP-TFII (\%) }\end{array}$ & $\chi^{2}$ & P-value \\
\hline Total samples & 80 & $32(40)$ & $48(60)$ & & 0.006 \\
\hline Age & & & & 0.02 & $>0.05$ \\
\hline$>50$ & 67 & $28(41.8)$ & $39(58.2)$ & & \\
\hline$<50$ & 10 & $5(50)$ & $5(50)$ & & \\
\hline Gender & & & & 1.52 & $>0.05$ \\
\hline Male & 52 & $25(48)$ & $27(52)$ & & \\
\hline Female & 25 & $8(32)$ & $17(68)$ & & \\
\hline Stage & & & & 0.55 & $>0.05$ \\
\hline $1-2$ & 15 & $6(40)$ & $9(60)$ & & \\
\hline $3-4$ & 37 & $19(51.4)$ & $18(48.6)$ & & \\
\hline Clinical stage & & & & 22.4 & $<0.01$ \\
\hline I-II & 11 & $3(27.3)$ & $8(72.7)$ & & \\
\hline III-IV & 61 & $29(47.5)$ & $32(52.5)$ & & \\
\hline
\end{tabular}

COUP-TFII, chicken ovalbumin upstream promoter transcription factor 2.
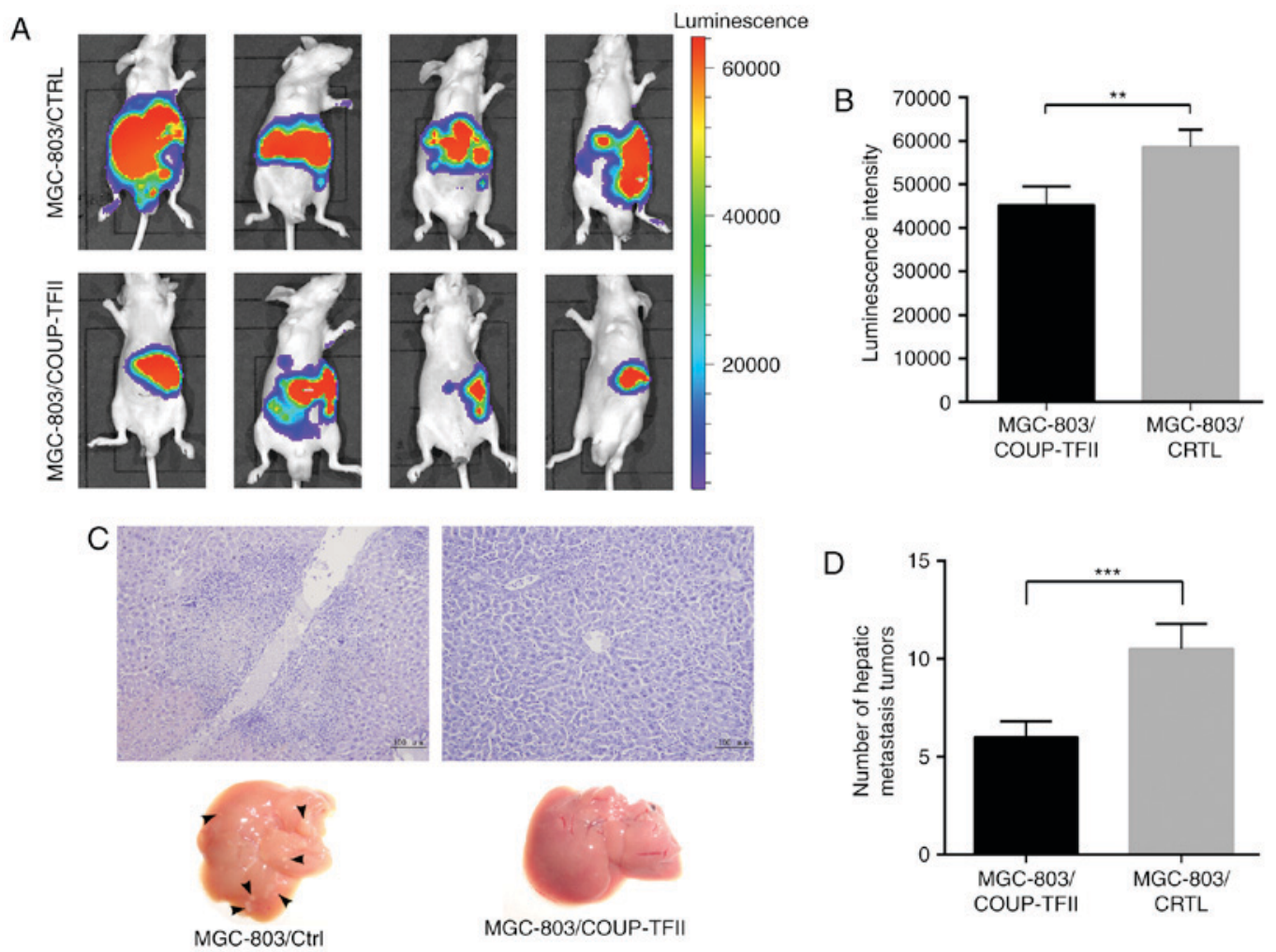

Figure 4. COUP-TFII inhibits tumor growth and hepatic metastasis of gastric cancer in vivo. (A) Bioluminescence imaging results of mice 4 weeks following the administration of MGC-803/COUP-TFII cells and mice injected with MGC-803 control cells. (B) Statistical analysis of luminescence intensity. (C and D) Number of hepatic metastases (black arrows) within the MGC-803/COUP-TFII group and the MGC-803 control group in nude mice tumor metastasis experiments in vivo $(\mathrm{P}<0.001)$. Scale bar, $100 \mu \mathrm{m} .{ }^{* * *} \mathrm{P}<0.01,{ }^{* * * *} \mathrm{P}<0.001$. COUP-TFII, chicken ovalbumin upstream promoter transcription factor 2.

are often abnormally expressed in breast cancer, prostate cancer and other cases of tumorigenesis (26). COUP-TFs are important members of the nuclear receptor family, and COUP-TFII, when isolated from the nuclear extracts of HeLa cells, can bind directly to the upstream promoter region of chicken ovalbumin in order to regulate its transcription (7). COUP-TFII has an important function in physiological development and tumorigenesis. In recent years, the involvement of COUP-TFII in malignant tumor progression has fast become a major focus of cancer research. 
Previous studies have revealed that numerous malignant tumor tissues abnormally express COUP-TFII, which regulates the invasion and migration of tumor cells (27). Qin et al (12) demonstrated that COUP-TFII inhibits the transforming growth factor $\beta$ (TGF- $\beta$ ) signaling pathway and promotes the growth and migration of prostate cancer cells. Furthermore, Qin et al (13) revealed that $~ 60 \%$ of prostate cancer samples had moderate to high expression levels of COUP-TFII; whereas only $5 \%$ of normal prostate tissues demonstrated abnormal expression of COUP-TFII. In addition, Bao et al (17) detected the expression of COUP-TFII in colon cancer tissue samples and revealed that the expression levels of COUP-TFII were elevated in cancerous tissues compared with normal mucosa tissues, and demonstrated that COUP-TFII could promote the invasion and migration of colorectal cancer cells via inhibition of the expression of adhesion molecules, such as tight junction protein 1, epithelial cadherin and $\beta$-catenin. Polvani et al (15) demonstrated that COUP-TFII was highly expressed in pancreatic cancer tissues. The invasion experiments demonstrated that the downregulation of COUP-TFII expression levels in pancreatic cancer cells may inhibit their invasiveness (15). However, there is evidence that COUP-TFII demonstrated specific expression patterns in certain tumor types $(16,28)$, such as in ovarian cancer, where low expression of COUP-TFII in stromal tissue, and abnormally high expression in epithelial tissues was detected, thus suggesting that COUP-TFII may have different roles among different tumor types.

In the present study, the expression of COUP-TFII in gastric cancer tissues was lower than that in the normal gastric mucosa, thereby suggesting that the expression of the COUP-TFII gene is reduced in gastric cancer tissues. This result is in contrast to previous studies demonstrating an increase in the expression of the COUP-TFII gene in prostate cancer (12), colon adenocarcinoma (17) and pancreatic cancer tissues (15). In order to investigate the effects of COUP-TFII on the proliferation and invasion of gastric cancer cells, an MGC-803 gastric cancer cell line with overexpressed COUP-TFII was established. The results of the MTT assay revealed that after 3 days of culture, the $490 \mathrm{~nm}$ relative absorbance of the MGC-803/COUP-TFII group was lower than that of the MGC-803 control group $(\mathrm{P}<0.05)$, thus suggesting that overexpression of COUP-TFII may significantly reduce the proliferative ability of gastric cancer cells. Furthermore, the results of the migration and invasion assays revealed that the migratory abilities of the MGC-803/COUP-TFII cells was significantly reduced compared with the MGC-803 control cells $(\mathrm{P}<0.001)$, thereby suggesting that overexpression COUP-TFII can decrease the mobility and invasive abilities of gastric cancer cells. In the present study, the COUP-TFII gene was knocked down in GES-1 cells. The MTT assay and the Transwell migration and invasion assays demonstrated that knockdown of COUP-TFII expression in GES-1 cells significantly increased cellular proliferation and the migratory and invasive abilities of the cells $(\mathrm{P}<0.01)$. These results suggest that the expression level of COUP-TFII can inhibit the proliferation, migration and invasion of gastric cancer cells, as well as function as a tumor suppressor gene.

Direct spread, lymph node metastasis, hematogenous metastasis and intraperitoneal implantation are the main

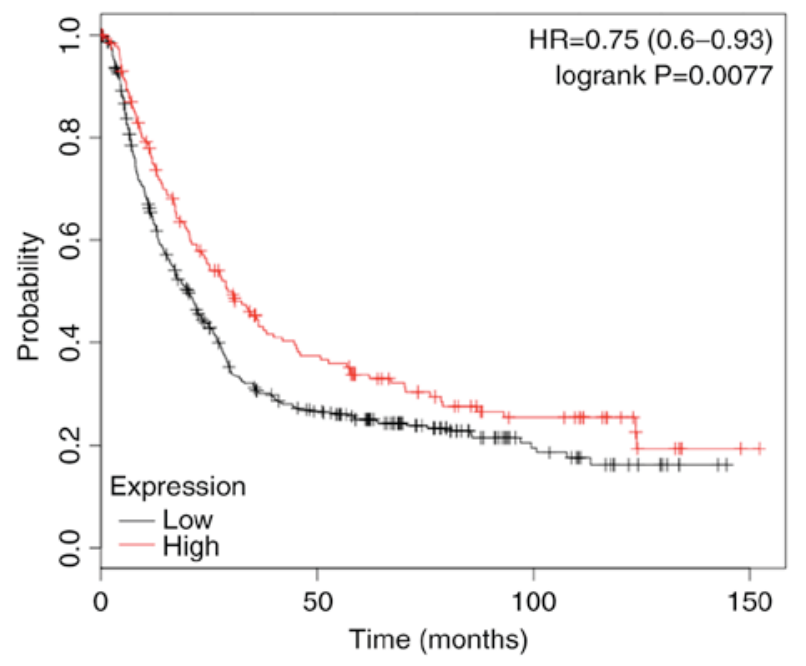

Number at risk

$\begin{array}{llll}\text { Low } 422 & 88 & 20 & 0 \\ \text { High } 171 & 52 & 21 & 1\end{array}$

Figure 5. Kaplan-Meier analysis of overall survival rates in patients with gastric cancer. HR, hazard ratio.

transfer pathways of gastric cancer, among which lymph node metastasis and hematogenous metastasis are the predominant pathways responsible for the development of distant metastasis of gastric cancer (29). Previous studies have revealed that COUP-TFII is involved in the regulation of vascular and lymph angiogenesis via the regulation of vascular endothelial growth factor as well as the expression level of its receptor, and thus acts as a regulator of neovascularization and lymph angiogenesis levels $(14,30,31)$. However, lymph node metastasis and hematogenous metastasis are complex processes and include multiple regulatory factors. Lin et al (32) demonstrated that COUP-TFII can affect levels of lymphangiogenesis in a breast cancer rat model via regulation of neuropilin 2 (NRP2) expression, thus suggesting that COUP-TFII may enact its therapeutic effect via regulation of NRP2 expression in order to affect the incidence of lymph node metastasis in breast cancer. The present study revealed that COUP-TFII expression in gastric carcinoma was reduced compared with normal gastric tissue, and that overexpression of COUP-TFII can downregulate the proliferation and migration of the MGC- 803 cell line. Furthermore, the results of present study suggest that COUP-TFII can inhibit the growth and invasion of gastric cancer and may also be involved in the development of distant metastases. Therefore, the present study use injection over expression of COUP-TFII stable cell strain in order to nude mice spleen in establishment of gastric cancer liver metastasis model by using in vivo imaging system (33). The results revealed that the number of liver metastases was lower in mice injected with COUP-TFII overexpressing gastric cancer cells compared with the control group $(\mathrm{P}<0.001)$, and hematoxylin and eosin analysis of liver metastasis tissue sections confirmed that in cases of gastric cancer, COUP-TFII can inhibit gastric cancer metastasis to the liver.

Furthermore, the results of the present study revealed that COUP-TFII can inhibit the growth and invasion of gastric cancer, as well as limit the occurrence of liver metastases. The grade, lymph node metastasis and distant metastasis 
are all factors considered in gastric cancer clinical stage classification. Database analysis of 80 patients with gastric cancer revealed that early stage (I-II) expression level of COUP-TFII was increased compared with expression levels of COUP-TFII in late stages (III-IV; $\mathrm{P}<0.01$ ), thus suggesting that COUP-TFII may influence the occurrence and development of gastric cancer. Clinical stages are closely associated with the prognosis of patients with gastric cancer (34). Furthermore, database analysis investigating survival rates demonstrated that increased expression of COUP-TFII significantly correlated with increased survival rates of patients with gastric cancer.

In conclusion, the results of the present study revealed that COUP-TFII was reduced in cases of gastric cancer. Furthermore, the results suggested that overexpression of COUP-TFII can inhibit growth, invasion and migration of gastric cancer in vitro and inhibit metastasis to the liver in vivo, and thus, may improve the survival rate of patients with gastric cancer. Therefore, the present study suggests that COUP-TFII may serve as a potential tumor biomarker and a target for molecular therapies. However, further studies aiming to uncover the underlying regulatory mechanisms of COUP-TFII associated with gastric cancer are required in order to determine the therapeutic potential of COUP-TFII. Future studies should investigate the association of COUP-TFII expression with regards to the TGF- $\beta$ /SMAD signaling pathway, the P53 signaling pathway, the mitogen-associated protein kinase signaling pathway and the epithelial-mesenchymal transition, in order to uncover the molecular mechanism underpinning the link between COUP-TFII and gastric cancer.

\section{Acknowledgements}

This study was supported by the Science and Technology Project of Natural Science Foundation of Fujian Province (grant nos. 2016J01639 and 2015J01564), the Medical Innovations Topic in Fujian Province (grant nos. 2016-CXB-8 and 2012-CXB-29) and Project of Xiamen Scientific and Technological Plan (grant no. 3502Z20134011).

\section{References}

1. Jemal A, Bray F, Center MM, Ferlay J, Ward E and Forman D: Global cancer statistics. CA Cancer J Clin 61: 69-90, 2011.

2. Jiang Y and Ajani JA: Multidisciplinary management of gastric cancer. Curr Opin Gastroenterol 26: 640-646, 2010.

3. Shi W, Wang J, Zhang M, Yu C, He W and Liu P: The multivariate prognostic analysis of 1340 gastric cancer patients who received radical resection. J Nanjing Med Univ (Nat Sci Ed) 31: 1310-1315, 2010.

4. Bray F, Jemal A, Grey N, Ferlay J and Forman D: Global cancer transitions according to the human development index (2008-2030): A population-based study. Lancet Oncol 13: 790-801, 2012.

5. Pastorcic M, Wang H, Elbrecht A, Tsai SY, Tsai MJ and O'Malley BW: Control of transcription initiation in vitro requires binding of a transcription factor to the distal promoter of the ovalbumin gene. Mol Cell Biol 6: 2784-2791, 1986.

6. Wang LH, Tsai SY, Cook RG, Beattie WG, Tsai MJ and O'Malley BW: COUP transcription factor is a member of the steroid receptor superfamily. Nature 340: 163-166, 1989.

7. Sagami I, Tsai SY, Wang H, Tsai MJ and O'Malley BW: Identification of two factors required for transcription of the ovalbumin gene. Mol Cell Biol 6: 4259-4267, 1986.
8. Miyajima N, Kadowaki Y, Fukushige S, Shimizu S, Semba K, Yamanashi Y, Matsubara K, Toyoshima K and Yamamoto T: Identification of two novel members of erbA superfamily by molecular cloning: The gene products of the two are highly related to each other. Nucleic Acids Res 16: 11057-11074, 1988.

9. Wang LH, Tsai SY, Sagami I, Tsai MJ and O'Malley BW: Purification and characterization of chicken ovalbumin upstream promoter transcription factor from HeLa cells. J Biol Chem 262: 16080-16086, 1987.

10. Wang LH, Ing NH, Tsai SY, O'Malley BW and Tsai MJ: The COUP-TFs compose a family of functionally related transcription factors. Gene Expr 1: 207-216, 1991.

11. Ladias JA and Karathanasis SK: Regulation of the apolipoprotein AI gene by ARP-1, a novel member of the steroid receptor superfamily. Science 251: 561-565, 1991.

12. Qin J, Wu SP, Creighton CJ, Dai F, Xie X, Cheng CM, Frolov A, Ayala G, Lin X, Feng XH, et al: COUP-TFII inhibits TGF- $\beta$-induced growth barrier to promote prostate tumorigenesis. Nature 493: 236-240, 2013.

13. Qin J, Chen X, Xie X, Tsai MJ and Tsai SY: COUP-TFII regulates tumor growth and metastasis by modulating tumor angiogenesis. Proc Natl Acad Sci USA 107: 3687-3692, 2010

14. Qin J, Chen X, Yu-Lee LY, Tsai MJ and Tsai SY: Nuclear receptor COUP-TFII controls pancreatic islet tumor angiogenesis by regulating vascular endothelial growth factor/vascular endothelial growth factor receptor-2 signaling. Cancer Res 70: 8812-8821, 2010.

15. Polvani S, Tarocchi M, Tempesti S, Mello T, Ceni E, Buccoliero F, D'Amico M, Boddi V, Farsi M, Nesi S, et al: COUP-TFII in pancreatic adenocarcinoma: Clinical implication for patient survival and tumor progression. Int J Cancer 134: 1648-1658, 2014.

16. Hawkins SM, Loomans HA, Wan YW, Ghosh-Choudhury T, Coffey D, Xiao W, Liu Z, Sangi-Haghpeykar H and Anderson ML: Expression and functional pathway analysis of nuclear receptor NR2F2 in ovarian cancer. J Clin Endocrinol Metab 98: E1152-E1162, 2013.

17. Bao Y, Gu D, Feng W, Sun X, Wang X, Zhang X, Shi Q, Cui G, Yu H, Tang $\mathrm{C}$ and Deng A: COUP-TFII regulates metastasis of colorectal adenocarcinoma cells by modulating Snaill. Br J Cancer 111: 933-943, 2014.

18. Edge SB and Compton CC: The American joint committee on cancer: The 7th edition of the AJCC cancer staging manual and the future of TNM. Ann Surg Oncol 17: 1471-1474, 2010.

19. Hu YM, Hu XP, Zhang L, Zhang SS and Xu JM: Uptake characteristics of levofloxacin for the eradication of Helicobacter pylori by GES-1 and MGC80-3 cells. Eur Rev Med Pharmacol Sci 20: 486-490, 2016.

20. Fan C,Lin Y,Mao Y,Huang Z, Liu AY, Ma H, Yu D, Maitikabili A, Xiao H, Zhang C, et al: MicroRNA-543 suppresses colorectal cancer growth and metastasis by targeting KRAS, MTA1 and HMGA2. Oncotarget 7: 21825-21839, 2016.

21. Zhang C, Zhang Y, Ding W, Lin Y, Huang Z and Luo Q: MiR-33a suppresses breast cancer cell proliferation and metastasis by targeting ADAM9 and ROS1. Protein Cell 6: 881-889, 2015.

22. Livak KJ and Schmittgen TD: Analysis of relative gene expression data using real-time quantitative PCR and the 2(-Delta Delta C(T)) method. Methods 25: 402-408, 2001.

23. Zhang Y, Xu G, Liu G, Ye Y, Zhang C, Fan C, Wang H, Cai H, Xiao R, Huang Z and Luo Q: miR-411-5p inhibits proliferation and metastasis of breast cancer cell via targeting GRB2. Biochem Biophys Res Commun 476: 607-613, 2016.

24. Rhodes DR, Kalyana-Sundaram S, Mahavisno V, Varambally R, Yu J, Briggs BB, Barrette TR, Anstet MJ, Kincead-Beal C, Kulkarni P, et al: Oncomine 3.0: Genes, pathways, and networks in a collection of 18,000 cancer gene expression profiles. Neoplasia 9: 166-180, 2007.

25. Rhodes DR, Yu J, Shanker K, Deshpande N, Varambally R, Ghosh D, Barrette T, Pandey A and Chinnaiyan AM: ONCOMINE: A cancer microarray database and integrated data-mining platform. Neoplasia 6: 1-6, 2004.

26. McKenna NJ and O'Malley BW: Combinatorial control of gene expression by nuclear receptors and coregulators. Cell 108: 465-474, 2002.

27. Navab R, Gonzalez-Santos JM, Johnston MR, Liu J, Brodt P, Tsao MS and Hu J: Expression of chicken ovalbumin upstream promoter-transcription factor II enhances invasiveness of human lung carcinoma cells. Cancer Res 64: 5097-5105, 2004.

28. Safe S, Jin UH, Hedrick E, Reeder A and Lee SO: Minireview: Role of orphan nuclear receptors in cancer and potential as drug targets. Mol Endocrinol 28: 157-172, 2014. 
29. $\mathrm{Xu} \mathrm{H}$ and $\mathrm{Xu} \mathrm{Y}$ : Recent advancement in metastasis of gastric cancer. Chin J Prac 31: 666-669, 2011.

30. Nagasaki S, Suzuki T, Miki Y, Akahira J, Shibata H, Ishida T, Ohuchi N and Sasano H: Chicken ovalbumin upstream promoter transcription factor II in human breast carcinoma: Possible regulator of lymphangiogenesis via vascular endothelial growth factor-C expression. Cancer Sci 100: 639-645, 2009.

31. Xu M, Qin J, Tsai SY and Tsai MJ: The role of the orphan nuclear receptor COUP-TFII in tumorigenesis. Acta Pharmacol Sin 36: 32-36, 2015.

32. Lin FJ, Chen X, Qin J, Hong YK, Tsai MJ and Tsai SY: Direct transcriptional regulation of neuropilin-2 by COUP-TFII modulates multiple steps in murine lymphatic vessel development. J Clin Invest 120: 1694-1707, 2010.
33. Yang S, Chen F, Zhang C, Gu X and Chen M: Establishment of liver metastasis model in nude mice with human gastric carcinoma. Beijing Lab Anim Sci 9: 13, 1992.

34. Shan F, Li Z, Bu Z, Zhang L, Wu A, Wu X, Wu Qi, Zong X, Li S, $\mathrm{Ji}$ X, Wu J and Ji J: The analysis of gastric cancer staging AJCC 6th edition and 7th edition. Chin J Prac Surg 31: 675-680, 2011.

(i) () () This work is licensed under a Creative Commons EY No No Attribution-NonCommercial-NoDerivatives 4.0 International (CC BY-NC-ND 4.0) License. 